\title{
VARIABILIDADE DE ATRIBUTOS QUÍMICOS DO SOLO SOB DIFERENTES GRIDS DE AMOSTRAGEM EM AGRICULTURA DE PRECISÃO
}

Tiago De Gregori Teixeira ${ }^{1}$, Telmo Jorge Carneiro Amado ${ }^{1}$, Rafael Pivotto Bortolotto ${ }^{1}$, Ademir de Oliveira Ferreira ${ }^{1}$, Diego Schmidt Schossler ${ }^{1}$, Paulo José Alba ${ }^{1}$, Fernando Dubou Hansel ${ }^{1}$

${ }^{1}$ Universidade Federal de Santa Maria - UFSM, Departamento de Solos, Santa Maria (RS). E-mail: rpbortolotto@yahoo.com.br

\section{RESUMO}

A agricultura de precisão vem sendo adotada por mais agricultores dia após dia, pela difusão da tecnologia entre empresas, produtores e técnicos. Com esses novos usuários da tecnologia surgem dúvidas técnicas sobre a correta utilização de suas ferramentas. Este trabalho apresenta um estudo sobre definição do grid de amostragem de solos; para isso foram analisados os grids 1 ha ponto ${ }^{-1}, 2$ ha ponto ${ }^{-1}, 3$ ha ponto $^{-1}$, e 5 ha ponto ${ }^{-1}$. Para os diferentes grids foram analisados fatores econômico (custo) e agronômico (atributos químicos de solo), a fim de melhor recomendação da aplicação de fertilizantes e corretivos. Com base nos diferentes grids de amostragem foi possível avaliar a variabilidade dos atributos químicos do solo. A análise agronômica é fator de alta influência na escolha do grid, pois compromete a qualidade dos dados conforme a quantidade de amostras diminui, devendo ser preconizada a aplicação de grids de amostragem menores possíveis. O tamanho grid em uma análise econômica é fator de baixa influência na escolha, pois a variação do custo do grid de 1 e 5 ha ponto ${ }^{-1}$ é de apenas $3,5 \%$.

Palavras-chave: grid de amostragem, análise econômica, taxa variada, variabilidade química, solo

\section{VARIABILITY OF SOIL CHEMICAL ATTRIBUTES UNDER DIFFERENT SAMPLING GRIDS IN PRECISION AGRICULTURE}

\begin{abstract}
The precision agriculture is being more adopted by farmers day after day, by diffusion of technology among companies, farmers and technicians. With these new users of technology technical questions arise about the correct use of its tools. This paper presents a study on the definition of grid soil sampling. One analyzed the grids: 1 soil sample per hectare, 1 soil sample per 2 hectares, 1 soil sample per 3 hectares and 1 soil sample per 5 hectares. For the different grids one analyzed agronomic (soil chemical properties) and economic (cost) factors, for better recommendation of fertilizer applications. Based on different sampling grids it was possible to assess the variability of soil chemical properties. Agronomic analysis is the factor of high influence in the choice of the grid, because it compromises the quality of data as the number of samples decreases and one should recommend to sampling grids as small as possible. In an economic analysis the choice of grid size, is a factor of low influence because the variation in the cost of a grid sample between 1 sample per 5 hectares to 1 per hectare is only $3.5 \%$.
\end{abstract}

Keywords: sampling grid, economic analysis, variable rate, chemical variability, soil 


\section{INTRODUÇÃO}

A eficiência em todos os setores da economia globalizada aumentou nos últimos anos, aumentando assim a competitividade. Para a agricultura não poderia ser diferente. A evolução da informática, tecnologias em geoprocessamento, sistemas de posicionamento global e muitas outras tecnologias estão proporcionando à agricultura uma nova forma de enxergar a propriedade, não observando de forma homogênea, e sim de forma heterogênea. Esta mudança está tornando cada vez mais o produtor rural em um empresário rural, por controlar cada vez mais sua produção (TSCHIEDEL \& FERREIRA, 2002).

Agricultura de precisão é um sistema de gerenciamento agrícola baseado na variação espacial de propriedades do solo e da planta encontrada nos talhões, visando à otimização do lucro, sustentabilidade e proteção do ambiente (MENEGATTI \& MOLIN, 2003). É uma ferramenta que vem sendo adotada a cada ano por mais produtores rurais que buscam racionalização dos insumos e maximização da produtividade.

O mapeamento da produtividade, proporcionado por monitores de colheita, destaca-se como alternativa moderna para gerenciar a variabilidade espacial e temporal de lavouras comerciais (MOLIN, 1997; MILANI et al., 2006), permitindo que sejam realizadas práticas de manejo de forma diferenciada dentro do mesmo talhão. A variabilidade espacial da produtividade pode ser reflexo de uma complexa interação de fatores, destacando-se aspectos fisiológicos da cultura, problemas referentes às intempéries climáticas e atributos do solo (MOLIN, 1997; SCHOSSLER et al., 2011)

A maneira como é realizada a amostragem de solo é um dos processos mais importantes, pois de nada vale uma análise química rigorosa e sofisticada de suas variáveis, se as amostras coletadas não são representativas do estudo realizado (CHUNG et al., 1995). A coleta de solo em grid busca identificar a variabilidade química existente em um talhão, para auxiliar o manejo da fertilidade do solo. $\mathrm{O}$ grid é a distribuição espacial dos pontos de coleta em um talhão, sendo cada ponto identificado por uma coordenada que possibilita a sua localização no campo (GIOTTO et al., 2004). A utilização desta ferramenta de agricultura de precisão, que é uma variável na maioria das vezes determinada por fatores empíricos, não é analisada econômica e agronomicamente.

A coleta de solo georreferenciada tem o objetivo de espacializar os atributos químicos do solo, em conjunto com ferramentas de sistema de informações geográficas (SIG) e geoestatística. Estas são utilizadas para geração de mapas que tornam visíveis a variabilidade química nas amostras do talhão. A escolha do grid de amostragem geralmente é determinada por fatores econômicos, pois quando o produtor contrata o serviço terceirizado, a cobrança do trabalho é relativa ao número de pontos coletados, não sendo realizada com base numa análise geoestatística prévia. $\mathrm{O}$ conhecimento se a área é homogênea ou heterogênea, quimicamente e texturalmente, será obtido após a análise do solo.

Áreas que possuem diferentes potenciais de rendimento devido a heterogeneidade necessitam de aplicações dos insumos de forma variável, na tentativa de homogeneizar a produtividade e/ou reduzir custos para consequentemente aumentar a rentabilidade da área (AMADO et al., 2007). Assim, a variabilidade espacial na produtividade das lavouras justifica $o$ manejo localizado como estratégia de uniformização do uso de insumos (AMADO et al., 2007).

Neste contexto, o objetivo deste trabalho foi avaliar a variabilidade de atributos químicos do solo sob diferentes 
grids de amostragem e sua influência na implementação da agricultura de precisão.

\section{MATERIAL E MÉTODOS}

O estudo foi realizado em 109,42 ha de área agrícola comercial do município de Rosário do Sul no estado do Rio Grande do Sul, coordenadas $30^{\circ} 19^{\prime} 39^{\prime \prime} \mathrm{S}$ e $55^{\circ} 02^{\prime}$ '26" O, utilizando o datum WGS84. A área possui altitude média de $120 \mathrm{~m}$, e o solo é classificado como argissolo vermelho distrófico típico (EMBRAPA, 2006). O sistema de manejo utilizado é o sistema plantio direto há 16 anos, sem nenhum histórico de intervenção com calcário. O clima da região é subtropical, temperado quente, com chuvas bem distribuídas e estações bem definidas (Cfa) (KÖPPEN, 1931). A declividade média da área é de 1,15 $\%$.

Avaliou-se a variabilidade espacial de atributos químicos utilizando diferentes grids de amostragem do solo, e análise econômica das atividades envolvidas. No experimento da variabilidade espacial dos atributos químicos do solo, a amostragem foi realizada no ano de 2010, seguindo uma malha de $100 \times 100 \mathrm{~m}$, gerada no software CR-Campeiro 7, totalizando 109 pontos de coleta. A localização dos pontos foi feita com a utilização de GPS de navegação modelo e-Trex Legend Garmin.

As coletas de solo foram realizadas manualmente com trado calador, sendo a amostra composta por 15 sub-amostras. As sub-amostras foram coletadas três no centro do ponto e mais 12 sub-amostras em um raio de $15 \mathrm{~m}$ da localização do ponto definido pelo GPS. As amostras foram coletadas na entrelinha, representando a camada de 0 $0,10 \mathrm{~m}$. A profundidade de coleta é recomendada pela CQFS-RS/SC (2004) para o sistema plantio direto consolidado.

Os atributos químicos avaliados foram: pH em água, índice SMP (método de análise e correção de acidez do solo, que se baseia no poder tampão do solo), teores de fósforo $(\mathrm{P})$ e de potássio $(\mathrm{K})$ extraídos por Mehlich. As determinações foram realizadas seguindo métodos descritos por Tedesco et al. (1995) no Laboratório de Manejo e Conservação do Solo da Universidade Federal de Santa Maria.

Os dados de fertilidade do solo dos grids de 2,3 e 5 ha ponto $^{-1}$, foram obtidos pela exclusão visual e sistemática dos pontos do grid inicial de 1 ha ponto $^{-1}$. O procedimento da exclusão dos pontos iniciou com o grid de 1 ha, onde teve $50 \%$ dos pontos excluídos, gerando com isso o grid de 2 ha. Para obtenção do grid de 3 ha foram retirados $67 \%$ dos pontos do grid inicial, a mesma metodologia foi utilizada para o grid de 5 ha, onde foram excluídos $81 \%$ dos pontos do grid de 1 ha (Figura 1).

A interpolação dos dados de atributos químicos do solo ocorreu no software CRCampeiro 7, sendo gerado os modelos digitais para os teores de $\mathrm{P}, \mathrm{K}$ e $\mathrm{pH}$ e índice SMP para os grids de 1 a 5 ha ponto $^{-1}$. Os parâmetros para geração dos modelos digitais no software foram: Grade $3 \times 3 \mathrm{~m}$, raio de busca 170, 240, 290 e $390 \mathrm{~m}$, para os grids de 1 ha, 2 ha, 3 ha e 5 ha ponto $^{-1}$ respectivamente.

Em um primeiro estudo foram avaliados os atributos químicos em diferentes locais de amostragens, e diferentes grids utilizando o coeficiente de variação, através de planilhas eletrônicas Excel. Os limites de coeficiente de variação (CV) utilizados no presente estudo foram baixo $(\mathrm{CV}<12 \%)$, médio $(12 \%<\mathrm{CV}<62 \%) \mathrm{e}$ alto $(\mathrm{CV}>62 \%)$ propostos por Warrick e Nielsen (1980). Para a realização da análise estatística foi utilizado o software de estatística Action, versão 2.1.101.228 para a realização do teste de Shapiro-Wilk. 

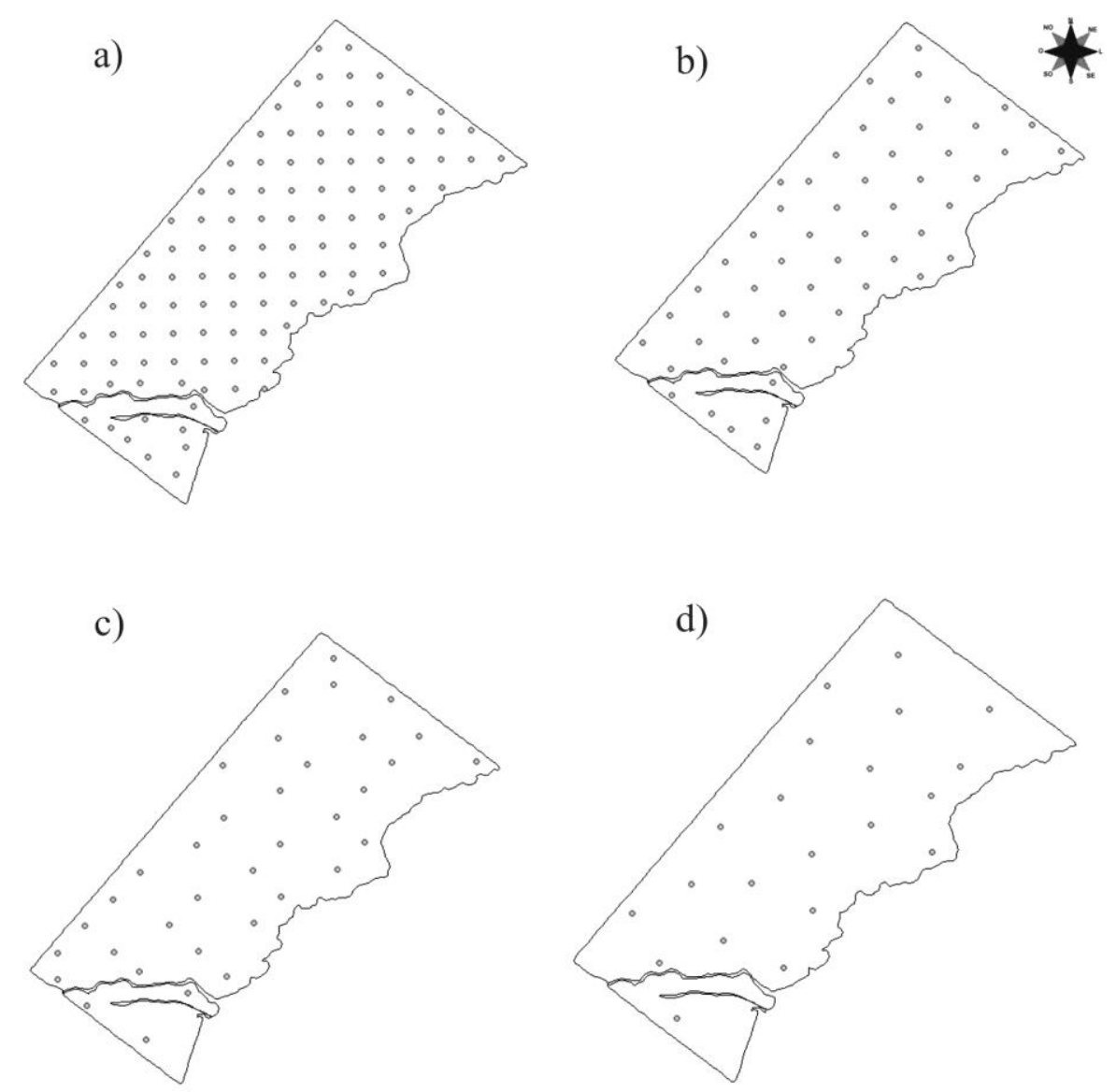

Figura 1. Espacialização dos pontos de coleta de solo; (a) grid 1 ha ponto ${ }^{-1}$ com 109 pontos amostrais; (b) grid 2 ha ponto ${ }^{-1}$ com 55 pontos amostrais; (c) grid 3 ha ponto ${ }^{-1}$ com 36 pontos amostrais; (d) grid 5 ha ponto ${ }^{-1}$ com 21 pontos amostrais.

$\begin{array}{rccc}\text { Em } & \text { um } & \text { segundo estudo foram } \\ \text { analisados } & \text { os } & \text { aspectos } & \text { agronômicos, }\end{array}$ juntamente com uma análise econômica dos grids utilizados para recomendação de fertilização.

Conforme descrito pela CQFSRS/SC (2004), adotou-se a busca de teores críticos altos de $\mathrm{K} 120 \mathrm{mg} \mathrm{dm}^{-3}$, e teores altos para $\mathrm{P}, 12 \mathrm{mg} \mathrm{dm}{ }^{-3}$, já $\mathrm{o} \mathrm{pH}$ considerado como ideal foi de valor 6. Para as expectativas dos teores, foram utilizadas as quantidades suficientes de produtos como superfosfato triplo $\left(48 \% \mathrm{P}_{2} \mathrm{O}_{5}\right)$, Cloreto de potássio $\left(60 \% \mathrm{~K}_{2} \mathrm{O}\right)$, e calcário com PRNT equivalente a $80 \%$.

O terceiro estudo foi a avaliação agronômica sobre a utilização dos grids, através da identificação de diferentes porcentagens em cada classe de atributo químico encontrada, destinado a representar espacialmente os teores encontrados conforme as Figuras 2, 3, 4 e 5.

\section{RESULTADOS E DISCUSSÃO}

Os teores de $\mathrm{P}$ (Figura 2) representados por "a" até "d" reduziram da classe de 3 a 6 , e 12 a $15 \mathrm{mg} \mathrm{dm}^{-3}$. Essa redução na classe dos teores de $\mathrm{P}$ reduz a área da classe de 3 a $6 \mathrm{mg} \mathrm{dm}^{-3}$ de 18,83 para 6,95 ha, diminuindo essa classe em quase 3 vezes. Já a classe dos teores médios, 6 a $9 \mathrm{mg} \mathrm{dm}^{-3}$ apresenta aumento de área de $128 \%$, passando de 31,19 para 71,11 ha. 
A variação espacial do atributo químico K (Figura 3), demonstra que a utilização de um grid maior, ou seja, mais hectares por um ponto de coleta de amostra remete a áreas mais homogêneas. As classes apresentadas sofrem pequenas variações conforme o grid utilizado, mas a análise visual dos mapas digitais mostra que há uma grande diferença na distribuição dessas classes nos mapas. Esta mesma variação foi encontrada para os atributos pH (Figura 4) e índice SMP (Figura 5).

A estatística descritiva dos atributos químicos do solo avaliados para os grids de 1 ha ponto $^{-1}, 2$ ha ponto $^{-1}, 3$ ha ponto $^{-1}$, e 5 ha ponto $^{-1}$, é apresentada na Tabela 1 . O valor encontrado de $\mathrm{CV}$ para $\mathrm{P}$ no grid de 5 ha foi $\mathrm{o}$ menor verificado, sendo uma variação pequena para os demais grids, ou seja $9 \%$ em relação ao grid de 3 ha que possui o maior $\mathrm{CV}$.

Como existe a variabilidade no solo, cada parte deve ser tratada conforme as suas necessidades, fazendo com que o produtor tenha o conhecimento detalhado de cada metro quadrado da sua propriedade (TSCHIEDEL \& FERREIRA, 2002).

Os resultados do CV para análise de $\mathrm{P}$ apresentam distribuição normal, segundo Shapiro-Wilk, sendo classificado para todos os grids analisados como médio $(12 \%<\mathrm{CV}<62 \%)$. A variabilidade para $\mathrm{P}$ também foi verificada por Corá \& Beraldo (2006). Souza et al. (1997) relataram CV de até $109 \%$ para $\mathrm{P}$.

O resultado referente ao atributo químico $\mathrm{K}$, apresentado para os grids analisados, mostra que o de 2 ha ponto $^{-1}$ apresenta os menores valores entre os demais referente a DP e CV, sendo demonstrado nos resultados do teste de Shapiro-Wilk.
Os resultados da análise para $\mathrm{pH}$, apresentaram baixo CV $(<12 \%)$ conforme encontrado por Cavalcante et al. (2007), que em seu estudo da variabilidade dos atributos químicos em diferentes sistemas, encontraram para o $\mathrm{pH}$ baixo $\mathrm{CV}$ para todas as situações. Já os resultados da análise do índice SMP, mostram que houve variação 0,01 no $\mathrm{CV}$ do grid 3 ha ponto $^{-1}$ em relação aos outros analisados.

Através do teste de Shapiro-Wilk (Tabela 1) foi possível confirmar que o índice SMP, não foi significativo para todos os grids, assim ratificando seu desvio padrão (DP) e CV menores que os outros atributos químicos. A existência de variabilidade espacial para esses atributos de solo, sobretudo aqueles relacionados a fertilidade e acidez, são evidências de que o manejo uniforme da adubação ou calagem, com doses de fertilizantes e corretivos baseadas em valores médios dos atributos, não é a decisão mais adequada, técnica e economicamente, de utilização de insumos em uma gleba agrícola (CORÁ et al., 2004; AMADO et al., 2009).

Diante dos resultados da análise econômica simplificada (Tabela 2) observase que mesmo a malha de pontos mais intensa, 1 ha ponto ${ }^{-1}$, não resultou em maior custo como era esperado, pois apresentou maior variabilidade dos seus atributos químicos, que refletiram em custo menor na utilização de fertilizantes e corretivos.

Analisando os custos totais do grid de 1 ha ponto ${ }^{-1}$ este foi $3,5 \%$ comparado com o de 5 ha ponto $^{-1}$. Os custos de uma tonelada de superfosfato triplo, e cloreto de potássio foram estimados, ambos, em US\$ 476,00. Já o custo da tonelada do corretivo de acidez do solo, o calcário, foi estimado em US\$ 47,00. $\mathrm{O}$ valor referente ao dólar foi a média do ano de 2011. 

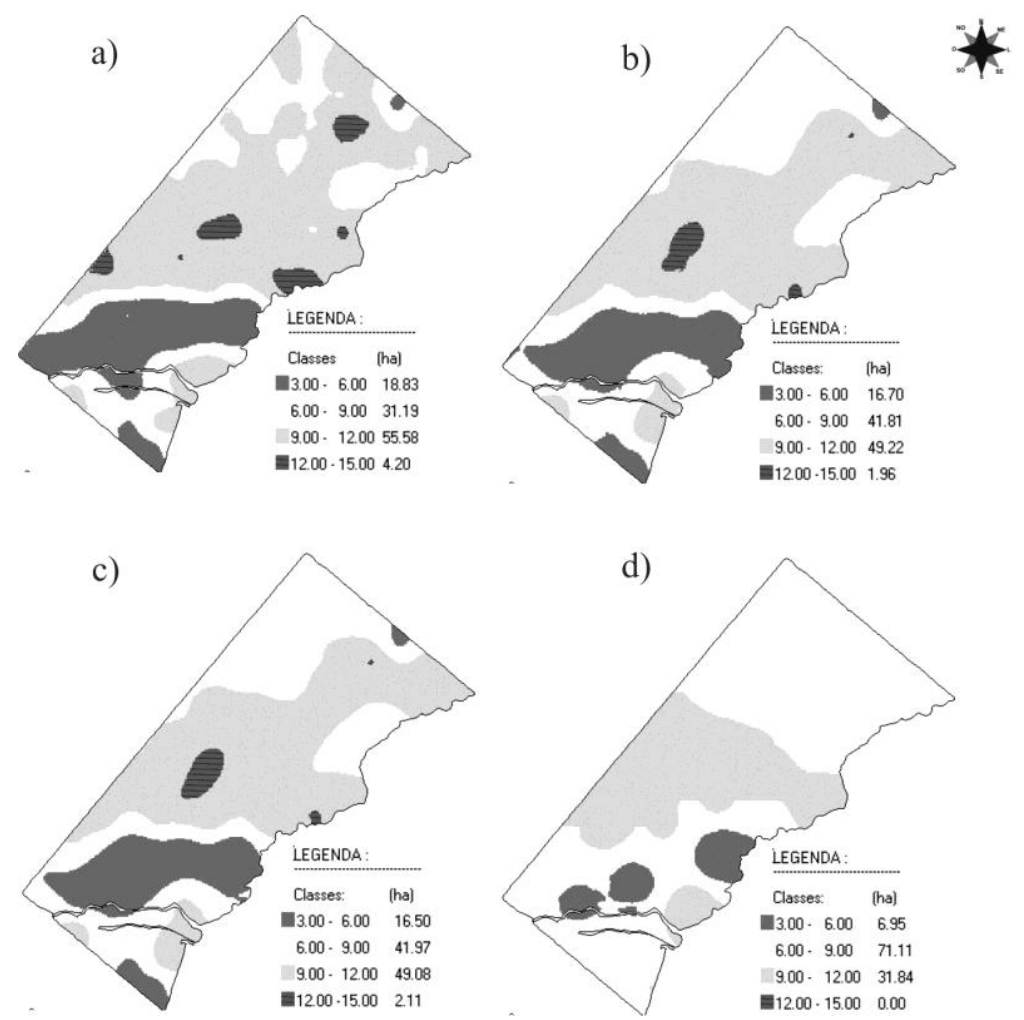

Figura 2. Modelos digitais dos diferentes grids para o atributo químico fósforo. (a) grid 1 ha ponto $^{-1}$; (b) grid 2 ha ponto $^{-1}$; (c) grid 3 ha ponto ${ }^{-1}$; (d) grid 5 ha ponto $^{-1}$.
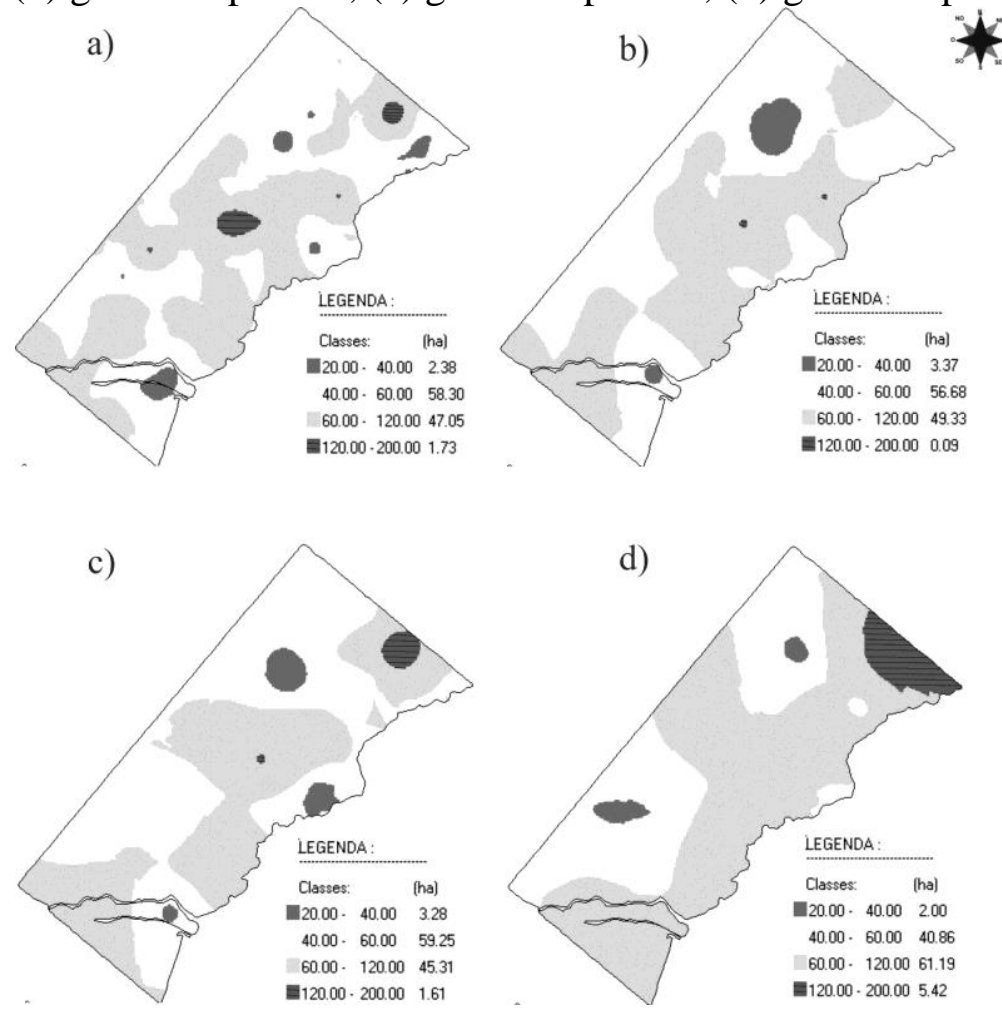

Figura 3. Modelos digitais dos diferentes grids para o atributo químico potássio. (a) grid tha ponto $^{-1}$; (b) grid 2 ha ponto ${ }^{-1}$; (c) grid 3 ha ponto $^{-1}$; (d) grid 5 ha ponto ${ }^{-1}$. 

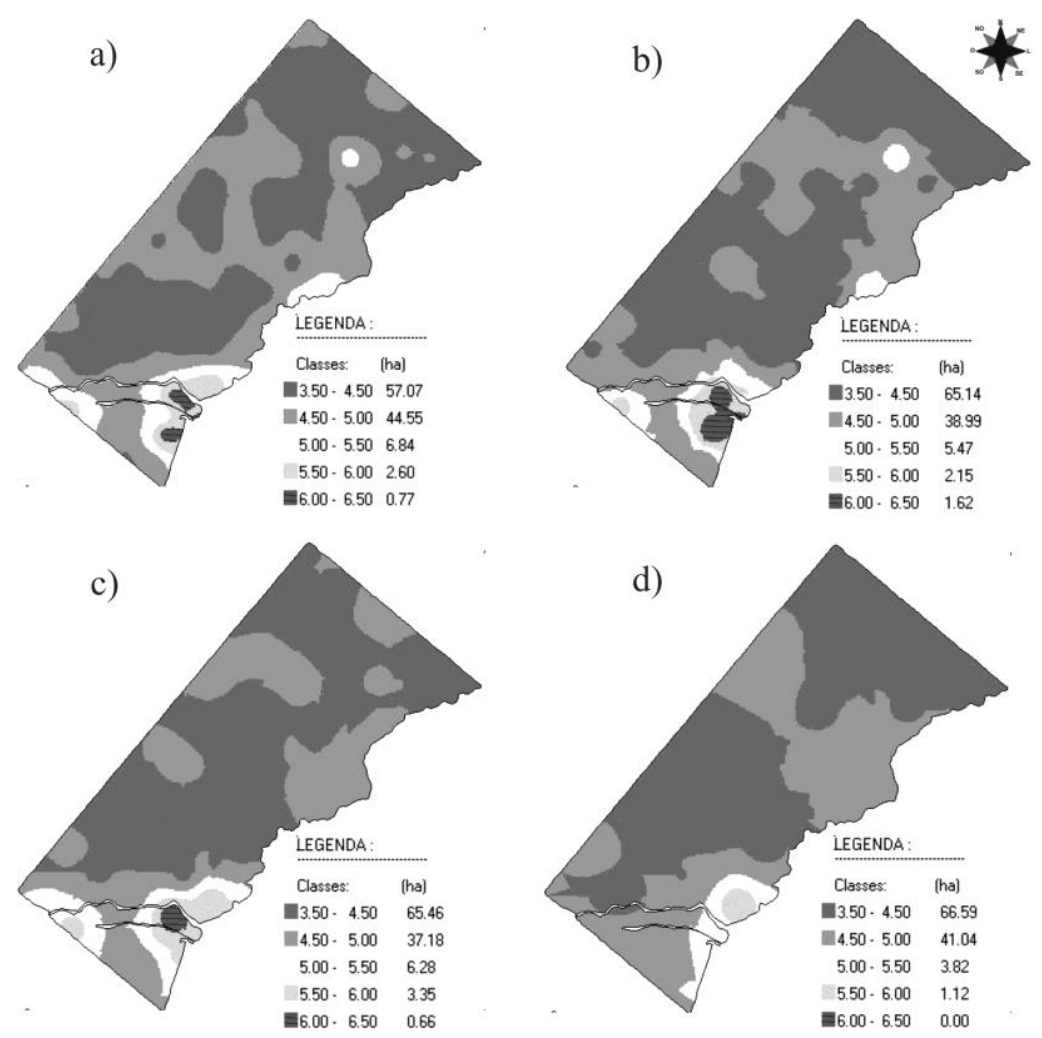

Figura 4. Modelos digitais dos diferentes grids para o atributo $\mathrm{pH}$. (a) grid 1 ha ponto ${ }^{-1}$; (b) grid 2 ha ponto $^{-1}$; (c) grid 3 ha ponto ${ }^{-1}$; (d) grid 5 ha ponto ${ }^{-1}$.
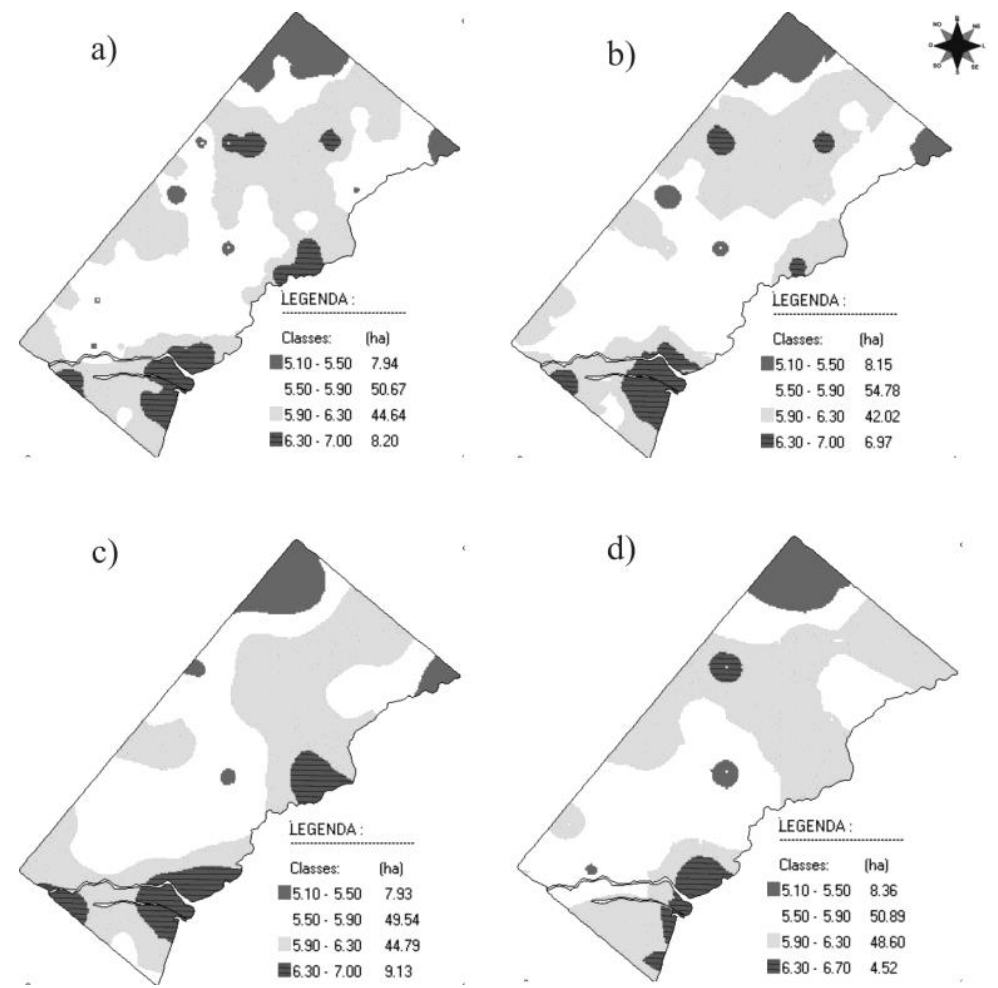

Figura 5. Modelos digitais dos diferentes grids para o índice SMP (a) grid 1ha ponto ${ }^{-1}$; (b) grid 2 ha ponto $^{-1}$; (c) grid 3ha ponto ${ }^{-1}$; (d) grid 5 ha ponto ${ }^{-1}$. 
Tabela 1. Parâmetros estatísticos dos atributos do solo avaliados para os diferentes grids, na camada de $0-0,10 \mathrm{~m}$.

\begin{tabular}{|c|c|c|c|c|c|c|c|c|}
\hline \multirow{2}{*}{ Grid } & \multirow{2}{*}{ Variável } & \multicolumn{7}{|c|}{ Parâmetro estatístico } \\
\hline & & Mínimo & Máximo & Mediana & Média & Desvio-Padrão & $\mathrm{CV}$ & Shapiro-Wilk \\
\hline \multirow{4}{*}{1 ha } & Fósforo & 2,98 & 13,68 & 9,43 & 8,74 & 2,62 & 0,30 & $0,94^{* * *}$ \\
\hline & Potássio & 28,00 & 177,60 & 58,00 & 62,14 & 23,32 & 0,38 & $0,76^{* *}$ \\
\hline & $\mathrm{pH} \mathrm{H} \mathrm{H}_{2} \mathrm{O}$ & 3,97 & 6,38 & 4,48 & 4,57 & 0,41 & 0,09 & $0,83^{* *}$ \\
\hline & Indice SMP & 5,15 & 6,96 & 5,86 & 5,90 & 0,36 & 0,06 & $0,98^{\mathrm{ns}}$ \\
\hline \multirow{4}{*}{2 ha } & Fósforo & 2,98 & 12,95 & 9,21 & 8,68 & 2,55 & 0,29 & $0,92^{* *}$ \\
\hline & Potássio & 28,00 & 127,20 & 57,20 & 60,00 & 19,41 & 0,32 & $0,85^{* *}$ \\
\hline & $\mathrm{pH} \mathrm{H} \mathrm{H}_{2} \mathrm{O}$ & 4,04 & 6,38 & 4,43 & 4,58 & 0,47 & 0,10 & $0,72^{* *}$ \\
\hline & Índice SMP & 5,15 & 6,96 & 5,81 & 5,90 & 0,38 & 0,06 & $0,96^{\mathrm{ns}}$ \\
\hline \multirow{4}{*}{3 ha } & Fósforo & 2,98 & 13,68 & 9,19 & 8,44 & 2,76 & 0,33 & $0,93^{*}$ \\
\hline & Potássio & 28,00 & 166,00 & 57,40 & 62,16 & 26,87 & 0,43 & $0,79^{* *}$ \\
\hline & $\mathrm{pH} \mathrm{H}{ }_{2} \mathrm{O}$ & 4,12 & 6,30 & 4,47 & 4,61 & 0,46 & 0,10 & $0,73^{* *}$ \\
\hline & Índice SMP & 5,15 & 6,96 & 5,80 & 5,90 & 0,42 & 0,07 & $0,98^{\mathrm{ns}}$ \\
\hline \multirow{4}{*}{5 ha } & Fósforo & 2,98 & 11,44 & 8,75 & 8,33 & 1,99 & 0,24 & $0,88^{*}$ \\
\hline & Potássio & 36,40 & 166,00 & 58,80 & 65,61 & 31,37 & 0,48 & $0,77^{* *}$ \\
\hline & $\mathrm{pH} \mathrm{H} \mathrm{H}_{2} \mathrm{O}$ & 4,12 & 5,72 & 4,45 & 4,54 & 0,34 & 0,07 & $0,80^{* *}$ \\
\hline & Índice SMP & 5,15 & 6,68 & 5,90 & 5,87 & 0,38 & 0,06 & $0,98^{\mathrm{ns}}$ \\
\hline
\end{tabular}

(1) Teste de Shapiro-Wilk para distribuição normal, significativo em níveis de $* * \mathrm{p} \leq 0,01 \mathrm{e}^{*} \leq 0,05$, ns: não significativo, respectivamente. Significativo indica que a hipótese para distribuição normal é rejeitada. Unidade das variáveis: Fósforo $\left(\mathrm{mg} \mathrm{dm}^{-3}\right)$, potássio $\left(\mathrm{cmolc} \mathrm{dm}^{-3}\right)$ e $\mathrm{pH} \mathrm{H}_{2} \mathrm{O}(1: 1)$.

Tabela 2. Análise econômica simplificada do custo por hectare dos diferentes grids e diferentes insumos utilizados.

\begin{tabular}{lcccccc}
\hline Grid & $\begin{array}{c}\text { Prestação } \\
\text { de serviço }\end{array}$ & $\begin{array}{c}\text { Superfosfato } \\
\text { triplo }\end{array}$ & $\begin{array}{c}\text { Cloreto de } \\
\text { Potássio }\end{array}$ & Calcário & Total & Área Total \\
\hline 1 ha & 29 & 52 & 233 & 130 & 445 & 48472 \\
2 ha & 27 & 54 & 236 & 134 & 451 & 49160 \\
3 ha & 24 & 54 & 235 & 129 & 442 & 48144 \\
5 ha & 21 & 57 & 215 & 135 & 429 & 46781 \\
\hline
\end{tabular}

US\$1 = R \$1,68A cotação do dólar foi calculada pela média histórica praticada no ano de 2011.

\section{CONCLUSÕES}

Com base nos diferentes grids de amostragem foi possível avaliar a variabilidade dos atributos químicos do solo.

A análise agronômica é fator de alta influência na escolha do grid, pois compromete a qualidade dos dados conforme a quantidade de amostras diminui.

O grid em uma análise econômica é fator de baixa influência na escolha do tamanho do grid, pois a variação do custo do grid de 1 e 5 ha ponto ${ }^{-1}$ é de apenas 3,5\%.

\section{REFERÊNCIAS BIBLIOGRÁFICAS}


AMADO, T.J,C; PERES, R.B; COSTA,J.A.; NICOLOSO, RS; TEIXEIRA, TG. 2007. A safra recorde analisada pelos mapas de rendimento no RS. Revista Plantio Direto, Passo Fundo, v.101, p.18-23, 2007.

AMADO, T.J.C.; PES, L.Z.; LEMAINSKI, C.L.; SCHENATO, R.B. 2009. Atributos químicos e físicos de Latossolos e sua relação com rendimentos de milho e feijão irrigados. Revista Brasileira de Ciência do Solo, Viçosa, v.33. p.831843.

CAVALCANTE, E.G.S.; ALVES, M.C.; SOUZA, Z.M.; PEREIRA, G.T. 2007. Variabilidade espacial de atributos químicos do solo sob diferentes usos e manejos. Revista Brasileira de Ciência do Solo, Viçosa, v.31, p.1329-1339.

CHUNG, C.K.; CHONG, S.K.; VARSA, E C. 1995. Sampling strategies for fertility on a stoy silt loam soil. Communications in Soil Science and Plant Analysis, New York, v.26. n.5-6, p.741-763.

CORÁ, J.E.; ARAUJO, A.V.; PEREIRA, G.T.; BERALDO, J.M.G. 2004. Variabilidade espacial de atributos do solo para adoção do sistema de agricultura de precisão na cultura da cana-de-açúcar. Revista Brasileira de Ciência do Solo, Viçosa, v.28, p.10131021.

CORÁ, J.E.; BERALDO, J.M.G. Variabilidade espacial de atributos do solo antes e após calagem e fosfatagem em doses variadas na cultura de cana-deaçúcar. Engenharia Agrícola, Jaboticabal, v.26, p.374-387, 2006.

CQFS-RS/SC - COMISSÃO DE QUÍMICA

E FERTILIDADE DO SOLO - RS/SC. Manual de adubação e de calagem para os Estados do Rio Grande do Sul e de Santa Catarina. Porto Alegre: Sociedade Brasileira de Ciência do Solo, 2004. 400p.
EMPRESA BRASILEIRA DE PESQUISA AGROPECUÁRIA - EMBRAPA. 2006. Sistema brasileiro de classificação de solos. Centro Nacional de Pesquisa de Solos, Rio de Janeiro, 306p.

GIOTTO, E.; ROBAINA, A.D.; SULZBACH, L. 2004. A agricultura de precisão como Sistema CR Campeiro 5. Santa Maria, Universidade Federal de Santa Maria, 330p.

KÖPPEN, W. 1931. Grundriss der Klimakunde. Berlin, Leipzig:Walter de Gruyter. 388p.

MENEGATTI, L. A.A.; MOLIN, J.P. 2003. Metodologia para identificação e caracterização de erros em mapas de produtividade. Revista Brasileira de Engenharia Agrícola e Ambiental, Campina Grande, v.7, n.2, p.367-374.

MILANI, L.; SOUZA, E.G. de; URIBEOPAZO, M.A.; GABRIEL FILHO, A.; JOHANN, J.A.; PEREIRA, J.O. 2006. Unidades de manejo a partir de dados de produtividade. Acta Scientiarum Agronomy, Maringá, v.28, p.591-598, 2006.

MOLIN, J.P. 1997. Agricultura de precisão, parte I: o que é e estado da arte em sensoriamento. Engenharia Agrícola, Jaboticabal, v.17, p.97-107.

SCHOSSLER, D.S.; AMADO, T.J.C.; SANTI, AL, TEIXEIRA, TG; GEIB, L.R.N. 2011. Fertilização potássica analisada economicamente com ferramentas de agricultura de precisão. Enciclopédia Biosfera, Goiânia, v.7, n.13, p. 595-603.

SOUZA, L.; COGO, N.P.; VIEIRA, S.R. 1997. Variabilidade de propriedades físicas e químicas do solo em um pomar cítrico. Revista Brasileira de Ciência do Solo, Viçosa, v.22, p.77-86.

TEDESCO, M.J.; GIANELLO, C.; BISSANI, C.A.; BOHNEN, H.; VOLKWEISS, S.J. 1995. Análises de solo, plantas e outros materiais. Porto 
Alegre, Universidade Federal do Rio Grande do Sul. 1995. 174p.

TSCHIEDEL, M.E. FERREIRA, M.F. 2002. Introdução à agricultura de precisão: conceitos e vantagens. Ciência Rural, Santa Maria, v.32, n.1, p.159-163.

Recebido em: 10/07/2012

Aceito para publicação em: 29/11/2013 